\title{
Diphenylphosphinoyl chloride as a chlorinating agent - the selective double activation of 1,2-diols $\uparrow$
}

\author{
David J. Fox, * Daniel Sejer Pedersen, Asger B. Petersen and Stuart Warren
}

\author{
Received 15th May 2006, Accepted 21st June 2006 \\ First published as an Advance Article on the web 6th July 2006 \\ DOI: $10.1039 / b 606881 b$
}

Treatment of 1,2-diols with diphenylphosphinoyl chloride in pyridine produces $\beta$-chloroethyl phosphinates which react with complete control of stereochemistry to give epoxides and azido-alcohols, useful intermediates in cyclopropane synthesis.

Stereochemically pure 1,2-diols, derived either from the catalytic asymmetric dihydroxylation of olefins ${ }^{1}$ or from other sources, are important intermediates in asymmetric synthesis. The hydroxy groups can be activated and differentially displaced by a variety of nucleophiles, often with high levels of regiocontrol due to adjacent electronic conjugating groups. Cyclic acylium ions, most often generated by reaction of diols with orthoesters ${ }^{2-4}$ or equivalents, ${ }^{5}$ can be ring opened with fluoride ${ }^{5}$ chloride ${ }^{2-4}$ and bromide to give vicinial halo-acylates. ${ }^{3}$ Along with cyclic carbonates, ${ }^{6}$ sulfites $^{7-9}$ and sulfates, ${ }^{10-12}$ chloro- and bromo-ethyl esters are valuable intermediates in the stereoselective synthesis of epoxides, ${ }^{6,13} \beta$ amino-alcohols $^{8,9,14}$ diamines $^{11}$ and amino-acids. ${ }^{7,10,15-17}$ In this paper we describe a new and simple method that provides not only the regioselective differentiation of 1,2-diols but also selective bis-activation.

During synthesis of cyclopropane-containing $\gamma$-amino ketones ${ }^{18}$ and esters ${ }^{19}$ the attempted bis-diphenylphosphinoylation of diols $\mathbf{1}$ and $\mathbf{2}$ with diphenylphosphinoyl chloride in pyridine resulted in inclusion of only one phosphinoyl group. Initially it was assumed that only one hydroxy group had reacted, but mass spectrometry and X-ray crystallography indicated that chlorine had replaced the hydroxy at the benzylic position with stereochemical inversion to give chloro-phosphinates 5 and 6 (Scheme 1). Methyl and diphenyl substituted diols ${ }^{13} \mathbf{3}$ and $\mathbf{4}$ also react selectively (Table 1). Only the reaction of diol 1 produced a small amount $(3 \%)$ of bisphosphinate product ${ }^{18} \mathbf{9}$. The reactions of diols $\mathbf{1}, \mathbf{2}$ and $\mathbf{3}$ produced only single stereo- and regioisomers of chloro-phosphinates.

The reaction of methyl cinnamate-derived diol ${ }^{17} \mathbf{1 0}$ produced a mixture of chloro-phosphinate regioisomers 11 and 12 along with bis-phosphinate 13 (Scheme 2). This result indicated that esters can also mediate the adjacent introduction of chlorine into 1,2diols. This was confirmed when non-aryl diol ${ }^{20} \mathbf{1 4}$ produced only the 2-Cl isomer of chloro-phosphinate $\mathbf{1 5}$.

X-Ray crystallography of chloro-phosphinate 6 (Scheme 1) showed that the introduction of chlorine occurred with inversion of stereochemistry, ${ }^{21}$ probably by $\mathrm{S}_{\mathrm{N}} 2$ reaction of chloride ion with the activated diol at the more activated position adjacent to either the aryl or ester groups. Hydrobenzoin $\mathbf{4}$ was chosen as a substrate to test the mechanism as it had substituted only once

University Chemical Laboratory, Lensfield Road, Cambridge, UK CB2 1EW.E-mail:djf34@cam.ac.uk

$\dagger$ Electronic supplementary information (ESI) available: experimental procedures and analytical details for new compounds and crystallographic data. See DOI: 10.1039/b606881b.

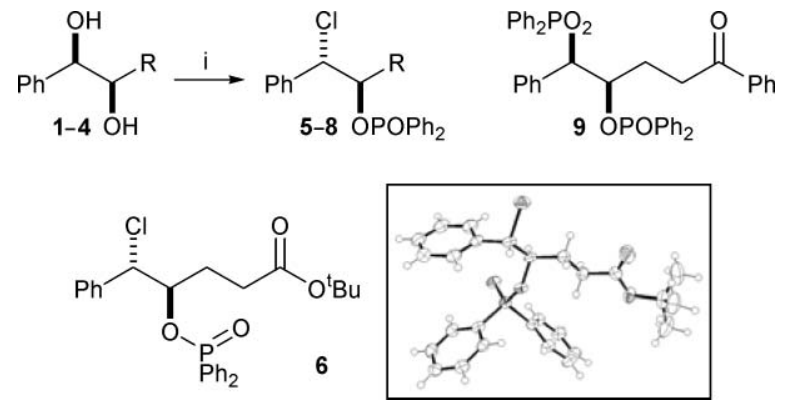

Scheme 1 Reagents and conditions: i) $\mathrm{Ph}_{2} \mathrm{POCl}$, pyridine, see Table 1. Inset: X-ray crystal structure of chloro-phosphinate $\mathbf{6}$ with thermal ellipsoids at $50 \%$ probability.

Table 1 Chloro-phosphinoylation of diols (see Scheme 1)

\begin{tabular}{llll}
\hline $\mathrm{R}$ & Diol & Chloro-phosphinate & Yield $(\%)^{a}$ \\
\hline$\left(\mathrm{CH}_{2}\right)_{2} \mathrm{COPh}$ & $\mathbf{1}$ & $\mathbf{5}$ & $66^{b}$ \\
$\left(\mathrm{CH}_{2}\right)_{2} \mathrm{CO}_{2}{ }^{\mathrm{t}} \mathrm{Bu}$ & $\mathbf{2}$ & $\mathbf{6}$ & 83 \\
$\mathrm{Me}$ & $\mathbf{3}$ & $\mathbf{7}$ & 60 \\
$\mathrm{Ph}$ & $\mathbf{4}$ & $\mathbf{8}$ & 71
\end{tabular}

${ }^{a}$ Isolated yield of chloro-phosphinate. ${ }^{b} 3 \%$ biphosphinate 9 also isolated.

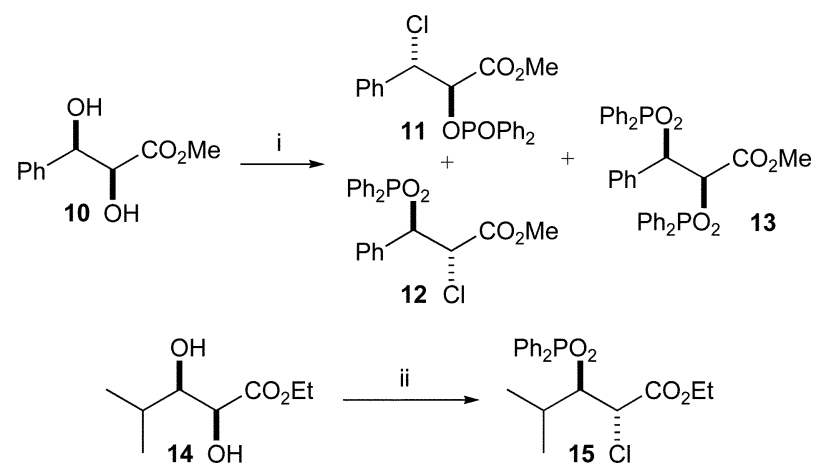

Scheme 2 Reagents and conditions: i) $\mathrm{Ph}_{2} \mathrm{POCl}$, pyridine, $11: 12: 13=$ $52: 17: 31$ (by ${ }^{1} \mathrm{H}$ NMR); ii) $\mathrm{Ph}_{2} \mathrm{POCl}$, pyridine, $45 \%$.

despite containing two benzylic alcohols. Two possible reaction pathways are either phosphinoylation of both alcohols followed by displacement of one phosphinate by chloride, or alternatively, reaction of the mono-phosphinate $\mathbf{1 6}$ with chloride, followed by a second phosphinoylation (Scheme 3). 


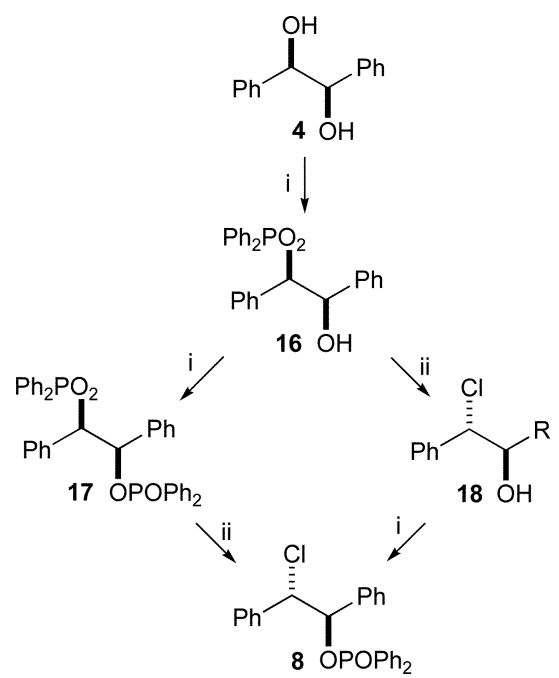

Scheme 3 i) Phosphinoylation; ii) $\mathrm{S}_{\mathrm{N}} 2$ diplacement by chloride.

The mono- and bis-phosphinates $\mathbf{1 6}$ and $\mathbf{1 7}$ were therefore synthesised from diol 4 to investigate their reactions with chloride ions in pyridine. Interestingly, neither the mono- nor bis-phosphinate reacts with pyridinium chloride, suggesting that these reactions do not take place in the chloro-phosphination reaction (Scheme 4). Unlike bis-phosphinate 17, the mono-phosphinate $\mathbf{1 6}$ does react with diphenylphosphinoyl chloride to give chloro-phosphinate $\mathbf{8}$. Finally, diol $\mathbf{4}$ does not react directly with pyridinium chloride.

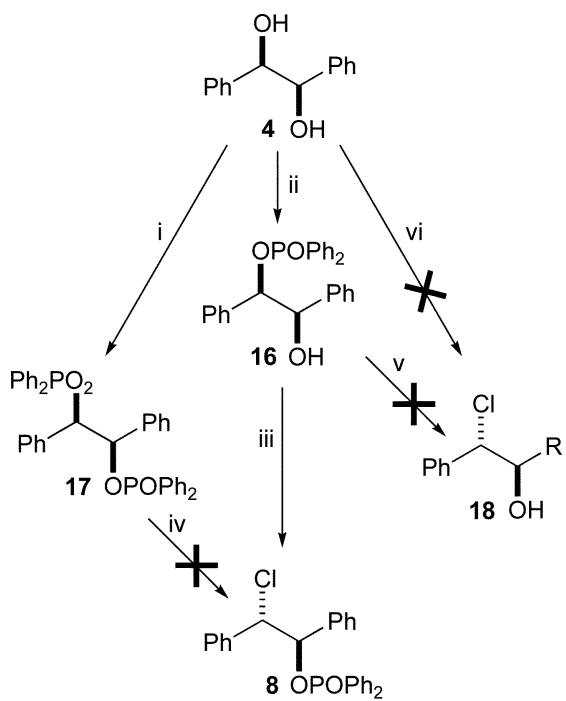

Scheme 4 Reagents and conditions: i) $\mathrm{Ph}_{2} \mathrm{POCl}, \mathrm{Et}_{3} \mathrm{~N}$, DMAP, THF, 36\%; ii) $\mathrm{Ph}_{2} \mathrm{POCl}, \mathrm{Et}_{3} \mathrm{~N}, \mathrm{DMAP}, \mathrm{CH}_{2} \mathrm{Cl}_{2}, 24 \%$; iii) $\mathrm{Ph}_{2} \mathrm{POCl}$, pyridine, $>95 \%$ (by ${ }^{1} \mathrm{H}$ NMR); iv) pyridine $\mathrm{HCl}$, pyridine, or $\mathrm{Ph}_{2} \mathrm{POCl}$, pyridine, $0 \%$; v) pyridine $\cdot \mathrm{HCl}$, pyridine, $0 \%$; vi) pyridine $\cdot \mathrm{HCl}$, pyridine, $0 \%$.

Given that mono-phosphinate $\mathbf{1 6}$ reacts with diphenylphosphinoyl chloride to give chloro-phosphinate $\mathbf{8}$, but that bisphosphinate $\mathbf{1 7}$ is not an intermediate, an alternative pathway to those suggested above must be sought. In addition, as chloride seems not to be nucleophilic enough to displace diphenylphosphinate in these reaction conditions, a more electrophilic intermediate must be involved. Cyclic phosphonium ion 20, formed via phosphinoylation of phosphorane $\mathbf{1 9}$, is suitably reactive (Scheme 5).<smiles>[R]C(O)C(Oc1ccccc1)C(=O)OC(C)C</smiles>

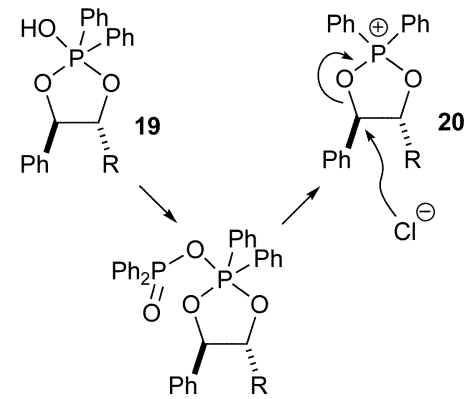

Scheme 5 Reagents and conditions: i) $\mathrm{Ph}_{2} \mathrm{POCl}$, pyridine.

Independent synthesis of phosphonium ion $\mathbf{2 0}$ and reaction with chloride ion was achieved via the reaction of diol $\mathbf{4}$ with $\mathrm{Ph}_{2} \mathrm{PCl}_{3}$ in pyridine (Scheme 6). ${ }^{22}$ Along with unreacted diol, the major product of the reaction is chloro-phosphinate 8. Peaks due to mono-phosphinate $\mathbf{1 6}$ can also be observed in the ${ }^{1} \mathrm{H}$ NMR spectrum of the crude reaction mixture. These products provide good evidence for the participation of phosphonium ion $\mathbf{2 0}$ in the chloro-phosphinoylation of diols with diphenylphosphinoyl chloride according to the mechanism proposed in Scheme 5.

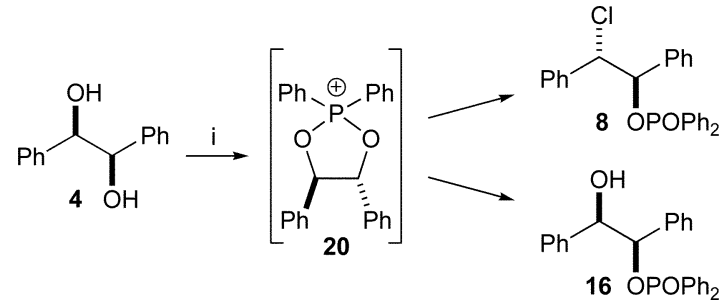

Scheme 6 Reagents and conditions: i) $\mathrm{Ph}_{2} \mathrm{PCl}_{3}$, pyridine $(4: 8: 16=43$ : $31: 26$, by ${ }^{1} \mathrm{H}$ NMR).

Finally, the reactions of the chloro-phosphinates were studied (Scheme 7). Displacement of the benzylic chlorides 5, 7 and 8 with azide produced mono-azide phosphinates $\mathbf{2 1 - 2 3}$ as single diastereoisomers; the phosphinate neither acts as a leaving group nor participates in the displacement of chloride. As with the related anti-azido-phosphinate, ${ }^{18}$ syn-azido-phosphinate 21 could be converted into mainly trans-cyclopropane 31. anti-Chlorophosphinates can also be converted into anti-epoxides 27 and 29: treatment with potassium carbonate in methanol ${ }^{13}$ results in removal of the diphenylphosphinate group ${ }^{23}$ and ring closure. The synthesis of cyclopropane ${ }^{24} \mathbf{3 0}$ results from the in situ basemediated reaction of epoxide 29. Overall, change in the order of reagents in the conversion of chloro-phosphinate $\mathbf{8}$ into azidoalcohols $\mathbf{2 6}$ and $\mathbf{2 8}$ reverses the stereochemistry of the final product. Overall reaction occurs with maintenance of stereochemistry via epoxide 27, but with inversion of stereochemistry at the benzylic position if the diphenylphosphinate is removed as the last step.

We hope to extend the simple chloro-phosphinoylation of 1,2diols to the synthesis of more complex and widely functionalised 


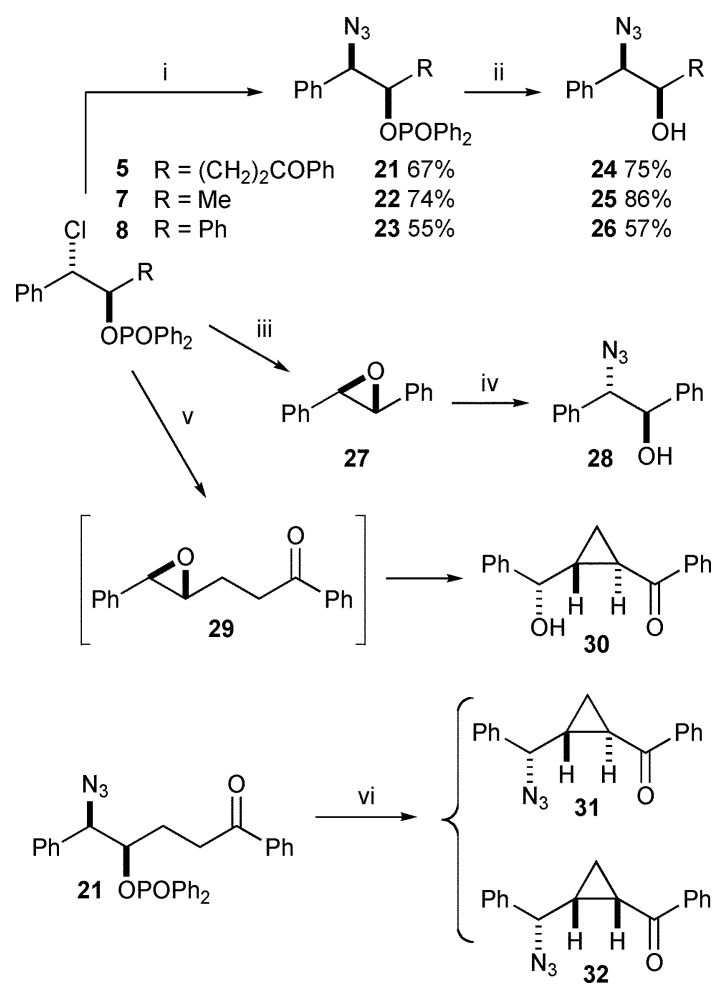

Scheme 7 Reagents and conditions: i) $\mathrm{NaN}_{3}, \mathrm{DMF}$; ii) $\mathrm{K}_{2} \mathrm{CO}_{3}, \mathrm{MeOH}$; iii) $\mathrm{K}_{2} \mathrm{CO}_{3}, \mathrm{MeOH}, 86 \%$; iv) $\mathrm{NaN}_{3}, \mathrm{DMF}, 75 \%$; v) $\mathrm{K}_{2} \mathrm{CO}_{3}, \mathrm{MeOH}, 79 \%$; vi) LDA, THF, $47 \%(\mathbf{3 1}: \mathbf{3 2}=9: 1)$.

molecules where the introduction of two different leaving groups with defined stereochemistry will have significant use.

\section{Acknowledgements}

We thank Dr John Davies for crystallography and the EPSRC for financial assistance towards the purchase of the Nonius CCD diffractometer. D. S. P. thanks the Alfred Benzon Foundation for financial support.

\section{References and notes}

1 H. C. Kolb, M. S. VanNieuwenhze and K. B. Sharpless, Chem. Rev., 1994, 94, 2483.

2 M. S. Newman and D. R. Olson, J. Org. Chem., 1973, 38, 4203.

3 M. S. Newman and C. H. Chen, J. Org. Chem., 1973, 38, 1173.

4 M. S. Newman and C. H. Chen, J. Am. Chem. Soc., 1973, 95, 278.

5 S. Hara and T. Fukuhara, US Pat. 2006/0014972, 2006.

6 H. T. Chang and K. B. Sharpless, J. Org. Chem., 1996, 61, 6456.

7 I. A. Sayyed and A. Sudalai, Tetrahedron: Asymmetry, 2004, 15, 3111 .

8 M. Braun, R. Fleischer, B. Mai, M. A. Schneider and S. Lachenicht, Adv. Synth. Catal., 2004, 346, 474.

9 K. Sahasrabudhe, V. Gracias, K. Furness, B. T. Smith, C. E. Katz, D. S. Reddy and J. Aube, J. Am. Chem. Soc., 2003, 125, 7914.

10 C. Y. Xiong, W. Wang and V. J. Hruby, J. Org. Chem., 2002, 67, 3514.

11 P. F. Richardson, L. T. J. Nelson and K. B. Sharpless, Tetrahedron Lett., 1995, 36, 9241.

12 B. B. Lohray, Y. Gao and K. B. Sharpless, Tetrahedron Lett., 1989, 30, 2623.

13 H. C. Kolb and K. B. Sharpless, Tetrahedron, 1992, 48, 10515.

14 H. T. Chang and K. B. Sharpless, Tetrahedron Lett., 1996, 37, 3219.

15 F. Soucy, L. Grenier, M. L. Behnke, A. T. Destree, T. A. McCormack, J. Adams and L. Plamondon, J. Am. Chem. Soc., 1999, 121, 9967.

16 J. G. Deng, Y. Hamada and T. Shioiri, J. Am. Chem. Soc., 1995, 117, 7824.

17 Z. M. Wang, H. C. Kolb and K. B. Sharpless, J. Org. Chem., 1994, 59, 5104.

18 D. J. Fox, S. Parris, D. S. Pedersen, C. R. Tyzack and S. Warren, Org. Biomol. Chem., 2006, DOI: 10.1039/b606874j.

19 D. J. Fox, D. S. Pedersen and S. Warren, Org. Biomol. Chem., 2006, DOI: $10.1039 / \mathrm{b} 606879 \mathrm{k}$

20 W. H. Pearson and M.-C. Cheng, J. Org. Chem., 1987, 52, 3176.

21 Crystal data for 6. $\mathrm{C}_{27} \mathrm{H}_{30} \mathrm{ClO}_{4} \mathrm{P}, M=484.93$, orthorhombic, space group $P 22_{1} 2_{1} 2_{1}, a=5.8203(10), b=11.4038(2), c=37.8022(9) \AA, a=$ $90, \beta=90, \gamma=90^{\circ}, U=2509.1(4) \AA^{3}, Z=4, \mu(\mathrm{Mo}-\mathrm{K} \alpha)=0.247 \mathrm{~mm}^{-1}$, 10165 reflections collected at 180(2) K using an Oxford Cryosystems Cryostream cooling apparatus, 4316 unique $\left(R_{\mathrm{int}}=0.056\right) ; R_{1}=0.053$, $w R_{2}=0.127[I>2 \sigma(I)]$, absolute structure parameter 0.02(10). CCDC reference number 600429. For crystallographic data in CIF or other electronic format see DOI: 10.1039/b606881b.

22 V. Chandrasekhar, T. Chivers, S. S. Kumaravel, A. Meetsma and J. C. van de Grampel, Inorg. Chem., 1991, 30, 3402.

23 D. J. Fox, D. S. Pedersen and S. Warren, Org. Biomol. Chem., 2006, DOI: $10.1039 / \mathrm{b} 606873 \mathrm{a}$

24 T. Boesen, D. J. Fox, W. Galloway, D. S. Pedersen, C. R. Tyzack and S. Warren, Org. Biomol. Chem., 2005, 3, 630. 